\section{Auf Röntgens Spuren}

Anlässlich des 174. Geburtstags von Wilhelm Conrad Röntgen eröffnete am 27. März 2019 in seinem Geburtshaus in Remscheid-Lennep eine Publikumsausstellung. Sie widmet sich dem Leben des Entdeckers der gleichnamigen Strahlen.

Ein inspirierender Ort der Informationsvermittlung und des gedanklichen Austauschs: Das Geburtshaus von Wilhelm Conrad Röntgen soll zukünftig als Treffpunkt für ein nationales wie internationales Publikum dienen. Einen ersten bedeutenden Schritt dahin bedeutet die neue Publikumsausstellung, die am 27. März 2019 im Beisein von Burkhard MastWeisz, Oberbürgermeister der Stadt Remscheid, und zahlreichen weiteren Ehrengästen aus Politik, Gesellschaft und Wissenschaft feierlich eröffnet wurde. Interessierte Bürgerinnen und Bürger hatten am 31. März 2019 erstmalig die Gelegenheit, die Ausstellung über das Leben von Röntgen zu besuchen. Die Einweihung des Geburtshauses ist für 2020 anlässlich des 125-jährigen Jubiläums der Entdeckung der Röntgenstrahlen und des 175. Geburtstags Röntgens geplant.

Die neue Publikumsausstellung im Röntgen-Geburtshaus, die maßgeblich von der NRW-Stiftung und dem Landschaftsverband Rheinland mitfinanziert wurde, widmet sich ganz der Person Röntgens und seiner Lebensleistung. Sie zeigt Stationen von seiner Geburt und seiner Familie in der bergischen Stadt Lennep, über seine Schulzeit, sein Studium bis hin zu seinen beruflichen Stationen in Gießen, Würzburg und München. Den Besucherinnen und Besuchern wird, angelehnt an die ursprüngliche Raumaufteilung um 1840, ein Rundgang durch vier Ausstellungsräume rund um das zentrale Treppenhaus ermöglicht. Sie können dabei die Geschichte dieses besonderen Hauses mit dem Wandel seiner Funktion und Nutzung im Laufe der Zeit nachvollziehen, die weltweiten Netzwerke Röntgens ergründen und sich in einer Schatzkammer auf Spurensuche mit Objekten aus seinem Nachlass begeben. Das Deutsche RöntgenMuseum stellt hierfür aus seinen Archiven eine Auswahl besonderer Dokumente, Urkunden und Briefe zur Verfügung. Virtuelle Medien erlauben zudem einfach und anschaulich den Einblick in zahlreiche weitere, vornehmlich handgeschriebene Doku-

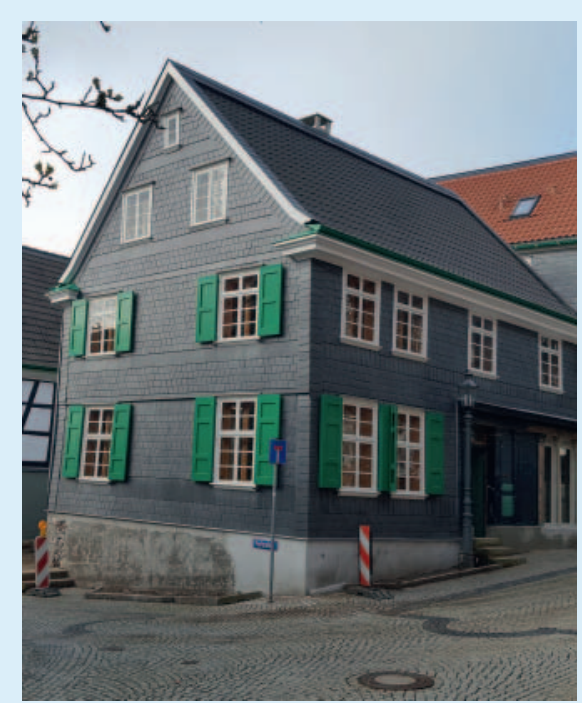

Das Röntgen-Geburtshaus in RemscheidLennep - mit neuen Fenstern und neuer Fassade. @: DRG.

mente. „Das Konzept konzentriert sich auf wenige Interventionen in den Räumen. Sie widmen sich ganz der Person Röntgens, seiner Biografie und seinen Netzwerken, lassen aber den authentischen Räumen noch Luft zum Atmen. “, erläutert Kurator Dr. Ulrich Hermanns vom Büro Dr. Ulrich Hermanns Ausstellung Medien Transfer GmbH. Das historische Ladenschaufenster ist bewusst als Blickfläche von außen gestaltet und bespielt, um bei Passanten Neugier und Interesse zu wecken. Für 2020 ist zudem ein gläserner Wintergarten geplant, der zusätzlich zur Publikumsausstellung Platz bieten soll für kleine Sonderausstellungen sowie öffentliche Veranstaltungen und Vorträge.

\section{Lebendiges Denkmal und inspirierender Ort}

Die Deutsche Röntgengesellschaft e. V. (DRG) hat im Jahr 2011 das Röntgen-Geburtshaus von der Stadt Remscheid erworben. Sie hat es sich zur Aufgabe gemacht, den Ursprungsort des weltberühmten Wissenschaftlers umfassend zu sanieren und so zu gestalten, dass er als ein lebendiges Denkmal und inspirierender Ort regional, national und international genutzt und erlebt werden kann. Während das benachbarte Deutsche Röntgen-Museum die wissenschaftliche Leistung von Röntgen in den

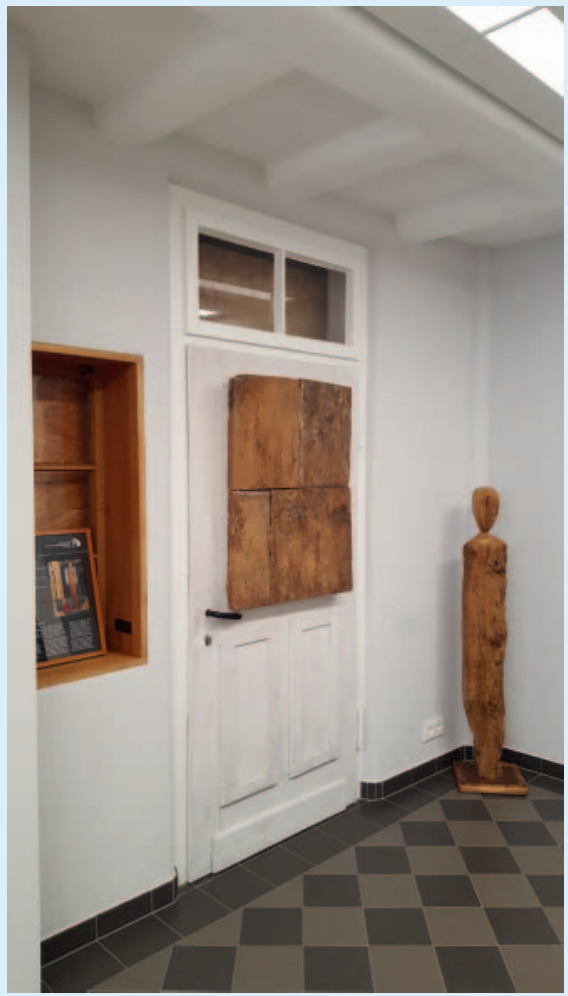

Im Inneren wurden Böden und Decken nach historischem Vorbild gestaltet. (): DRG.

Fokus rückt und bereits zahlreiche Angebote für die breite Öffentlichkeit etabliert hat, die zum kreativen Forschen und erlebnisreichen Entdecken einladen, soll das Geburtshaus künftig für ein zusätzliches und andersartiges Angebot stehen: dem emotionalen Moment der Begegnung in einer speziellen Atmosphäre. Für die breite Öffentlichkeit meint dies die Begegnung mit dem Haus in seiner historischen Bedeutung sowie mit der Biografie von Röntgen, die vielfältige Verknüpfungen offenbart und zahlreiche Identifikationsmöglichkeiten bietet. Dr. Uwe Busch, Direktor des Deutschen Röntgen-Museums: „Zusammen mit dem Deutschen Röntgen-Museum und dem dort ebenfalls eingerichteten „Röntgen-Labor“ für Schüler, Studenten und Auszubildende verleiht das Geburtshaus Remscheid-Lennep ein authentisches Gesicht als Röntgenstadt und sorgt mit einem vielfältigen Informations- und Bildungsangebot für hohe touristische Attraktivität im Bergischen Dreieck. In Kooperation mit der Stadt Remscheid und dem Museum wird das Geburtshaus außerdem zukünftig auch eine Schlüsselrolle als Begegnungsort im Rahmen der jährlich stattfindenden Verleihung der Röntgen-Plakette einnehmen." 


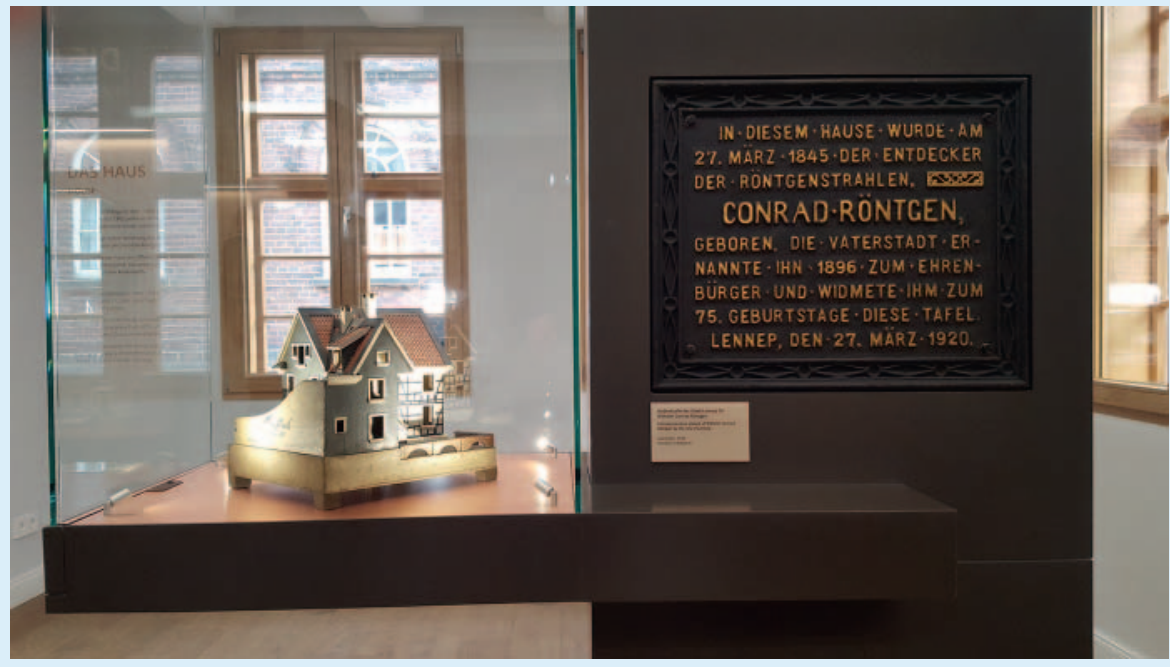

Die Ausstellung zeigt auch ein historisches Modell des Geburtshauses von Wilhelm Conrad Röntgen, sowie eine Originalplakette von 1920. @: DRG.

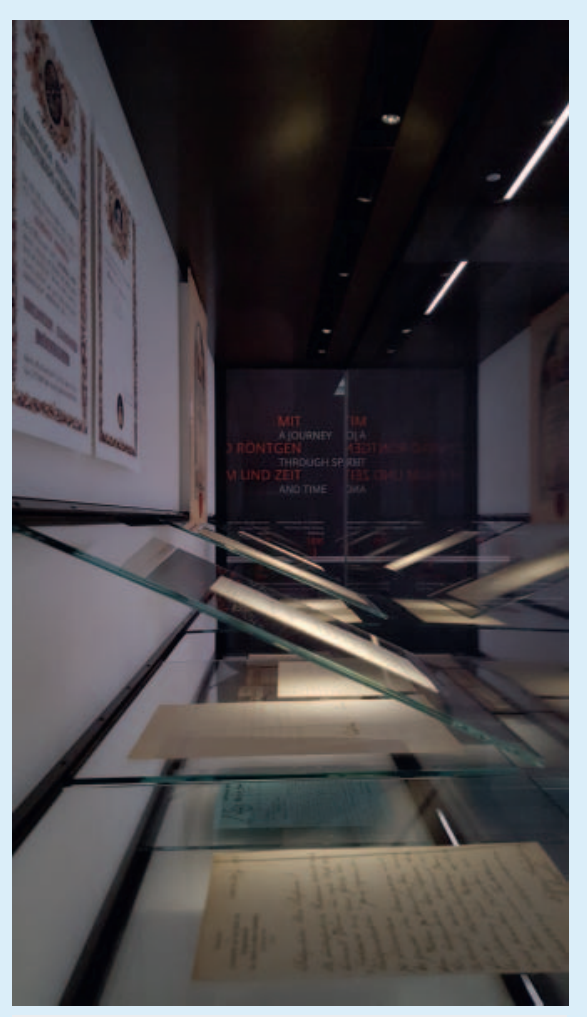

In der Schatzkammer können die Besucherinnen und Besucher Originaldokumente, z. B. Briefe an Röntgen von herausragenden Persönlichkeiten, entdecken. @: DRG.
Für die Fachöffentlichkeit, insbesondere aus (Natur-)Wissenschaft und Forschung, bietet sich künftig mit dem Geburtshaus ein einzigartiger Treffpunkt für den fachlich motivierten Austausch sowie eine außergewöhnliche, inspirierende Arbeitsumgebung für neue Forschergenerationen. Auch die DRG hat das Geburtshaus als eine zentrale Anlaufstelle fest eingeplant: „Zukünftig werden im Geburtshaus nicht nur Gremiensitzungen der DRG, von assoziierten Fachgesellschaften und Partnern sowie Workshops und Kurse stattfinden. Wir wollen hier auch gemeinsam mit Partnern ein zentrales „International Roentgen Training and Testing Center (iRTTC)“ einrichten und so zukünftig Intensivworkshops, Simulationskurse und Zertifizierungsprüfungen im Geburtshaus bündeln“, erläutert Prof. Dr. Stefan Schönberg, Präsident der DRG.

\section{Breite nationale wie internationale Unterstützung}

Getragen, begleitet und finanziert wird das Geburtshaus von den Mitgliedern der DRG sowie zahlreichen Bürgerinnen und Bürgern und Unternehmen aus der Region. Zu den rund 200 Partnern, Förderern und Spendern gehören u. a. Bracco Imaging Deutschland,

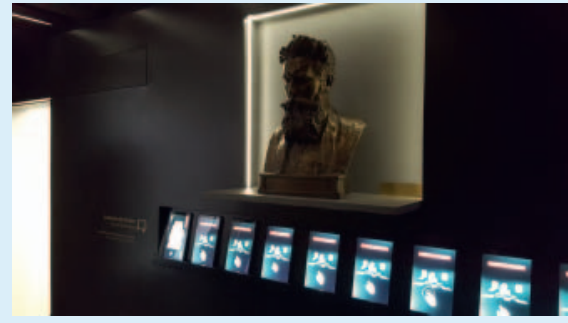

Per Tablet lässt sich mehr über die Originaldokumente erfahren. @: DRG.

die Dr. Wolf, Beckelmann und Partner $\mathrm{GmbH}$, die Radiological Society of North America und der Georg Thieme Verlag. Die Planung und Umsetzung erfolgt in enger Kooperation mit dem Deutschen RöntgenMuseum und der Stadt Remscheid sowie unter Einbindung lokaler Dienstleister.

\section{Zentraler Bestandteil des Jubiläumsjahrs 2020}

Am 8. November 1895 entdeckte Prof. Dr. Wilhelm Conrad Röntgen im Physikalischen Institut der Julius-Maximilians-Universität Würzburg die nach ihm benannten Strahlen, denen er zunächst den Namen X-Strahlen gab. Seine Entdeckung einer völlig neuen Art von Strahlen jährt sich 2020 zum 125sten Mal. Der am 27. März 1845 in Remscheid-Lennep geborene erste Nobelpreisträger für Physik würde im Röntgenjahr 2020 seinen 175. Geburtstag begehen. Um dieses bedeutsame Kapitel deutscher Wissenschaftsgeschichte einem Fachpublikum und der breiten Öffentlichkeit nahe zu bringen, haben sich Universitäten, Hochschulen, Städte, Gesellschaften, Verbände und Institutionen zusammengeschlossen, die sich dem Erbe Wilhelm Conrad Röntgens verpflichtet fühlen und gemeinsam das Jahr 2020 als „Röntgen-Jahr“ gestalten wollen. Dem Geburtshaus von Wilhelm Conrad Röntgen kommt hierbei eine tragende Rolle zu. Seine feierliche Eröffnung ist für den 27. März 2020 geplant.

Mehr Informationen:

www.roentgen-geburtshaus.de 